Gynäkologische Endokrinologie 2013 .

11:140-143

DOI 10.1007/s10304-013-0553-7

Online publiziert: 21. März 2013

๑) Springer-Verlag Berlin Heidelberg 2013
Epidemiologische Studien belegen ein erhöhtes kardiovaskuläres Risiko für Frauen nach der Menopause [9]. Ein Zusammenhang mit dem menopausenbedingten Östrogenabfall wird diskutiert $[15,21]$. Der Einfluss einer Östrogentherapie auf das Herz hängt jedoch vom Zeitpunkt der hormonellen Intervention ab. Östrogene haben eine vorteilhafte, weil vasodilatative, antiinflammatorische und antiatherosklerotische Wirkung, wenn sie auf gesunde oder geringfügig atherosklerotisch veränderte Koronararterien treffen. Neutral oder nachteilig, weil proinflammatorisch und plaquedestabilisierend ist ihr Einfluss hingegen, wenn die Koronararterien fortgeschritten atherosklerotisch sind [14]. Als günstiges Zeitfenster für den Beginn einer Hormonersatztherapie (HRT) werden die ersten 6-10 Jahre nach der Menopause vermutet [8].

\section{》) Die Wirkung einer Östrogentherapie auf das Herz hängt vom Zeitpunkt der Intervention ab}

Auch die Aktivität des autonomen Nervensystems (ANS) des Herzens beeinflusst die Entwicklung einer koronaren Herzkrankheit (KHK; [12]). Das ANS kann beispielsweise anhand der Herzfrequenzvariabilität (HRV) beurteilt werden. Die Frage ist, in welchem Zusammenhang die HRV mit der endo- und exogenen Östrogenexposition steht, ob es also eine Verbindung zwischen dem postmenopausalen Östrogenmangel, der HRVVeränderung und einer KHK gibt und in-

G. Capaldo ${ }^{1} \cdot$ M. Wilhelm ${ }^{2} \cdot$ P. Stute ${ }^{1}$

${ }^{1}$ Abteilung für Gynäkologische Endokrinologie und Reproduktionsmedizin, Frauenklinik, Inselspital Bern, Bern

${ }^{2}$ Kardiovaskuläre Prävention und Rehabilitation, Universitätsklinik für Kardiologie, Inselspital Bern, Bern

\title{
Herzfrequenzvariabilität in der Postmenopause
}

wiefern eine Östrogentherapie die HRV und hierüber das KHK-Risiko beeinflusst.

\section{Herzfrequenzvariabilität}

Der Sinusknoten des Herzens erzeugt einen eigenen konstanten Rhythmus, der für den Sinusrhythmus verantwortlich ist. Um auf verschiedene innere und äußere Reize reagieren zu können, wird die Herzfrequenz hauptsächlich durch das kardiale ANS beeinflusst, was in einer Variabilität der Herzfrequenz resultiert. Weitere Faktoren, die die HRV beeinflussen, sind humorale Systeme, die Temperaturregulation, Hormone und der zirkadiane Rhythmus. Die HRV kann in einzelne Frequenzspektren aufgespalten und einzelnen Einflussfaktoren zugeordnet werden [25]. Eine Übersicht über die hier verwendeten HRV-Parameter und ihre phy- siologische Bedeutung ist in $\bullet$ Tab. 1 gegeben.

Durch eine beschleunigte Depolarisation in der Diastole fördert eine Sympathikusstimulation den automatischen intrinsischen Sinusrhythmus, während der Parasympathikus das Gegenteil bewirkt und durch Hyperpolarisation die Diastole verlängert [2]. Eine Prädominanz des Sympathikotonus mit verminderter vagaler Aktivität führt zu einer verminderten $H R V$ und kann mit einer kardialen elektrischen Instabilität assoziiert sein [26]. Bei Patienten mit stattgehabtem Myokardinfarkt geht eine erniedrigte HRV mit einem erhöhten Risiko des plötzlichen Herztods einher. Eine niedrige HRV kann auch für eine subklinische kardiale Erkrankung stehen [29]. Auch bei verschiedenen nichtkardialen Erkrankungen wie dem Diabetes mellitus kann das

\begin{tabular}{|c|c|}
\hline SDNN & $\begin{array}{l}\text { Standardabweichung der Normal-to-normal(NN)-Intervalle; steht für die allgemeine } \\
\text { Variabilität der Herzfrequenz („,total power“) und wird durch den Sympathikus und } \\
\text { Parasympathikus beeinflusst }\end{array}$ \\
\hline RMSSD & $\begin{array}{l}\text { Quadratwurzel der Differenzen zwischen aufeinanderfolgenden NN-Intervallen; kann } \\
\text { die respiratorische Sinusarrhythmie erfassen und ist ein Marker des Vagotonus }\end{array}$ \\
\hline HF & $\begin{array}{l}\text { „High frequency power“; steht für Herzfrequenzfluktuationen von } 0,15-0,4 \mathrm{~Hz} \text { und } \\
\text { wird vom Parasympathikus beeinflusst. Eine hohe HF ist ein Marker einer hohen Para- } \\
\text { sympathikusaktivität. }\end{array}$ \\
\hline LF & $\begin{array}{l}\text { „Low frequency power“; steht für Herzfrequenzfluktuationen von } 0,04-0,15 \mathrm{~Hz} \text {; wird } \\
\text { sowohl vom Parasympathikus als auch vom Sympathikus beeinflusst und reflektiert die } \\
\text { Barorezeptorfunktion. Nach einem Orthostasetest zeigen die „LF power“ und adrener- } \\
\text { ge Aktivität eine gute Korrelation. }\end{array}$ \\
\hline LFnu & LF in normalisierten Einheiten \\
\hline HFnu & HF in normalisierten Einheiten \\
\hline $\begin{array}{l}\text { LF/HF- } \\
\text { Verhältnis }\end{array}$ & $\begin{array}{l}\text { Ist insbesondere nach einem Orthostasetest ein Marker der sympathovagalen Balance. } \\
\text { Höhere Werte sprechen für eine stärkere adrenerge Aktivierung. }\end{array}$ \\
\hline TP & $\begin{array}{l}\text { „Total power“; steht für die allgemeine Variabilität der Herzfrequenz, herbeigeführt } \\
\text { durch alle Einflussfaktoren }\end{array}$ \\
\hline
\end{tabular}


sympathovagale Gleichgewicht gestört und die absolute HRV erniedrigt sein.

\section{Herzfrequenzvariabilität in verschiedenen endokrinen Lebensphasen}

Die Hormonschwankungen im Rahmen eines normalen Menstruationszyklus scheinen sich auch auf die elektrische Stabilität des Herzens auszuwirken, denn die Prävalenz von Arrhythmien ist in der Lutealphase gegenüber der Follikelphase deutlich erhöht [22]. Dies spiegelt sich auch in der HRV wider, wobei zyklusabhängige Veränderungen der HRV mehrfach beschrieben wurden $[1,23,24$, 31]. In Kurzzeitmessungen zeichnete sich während der Lutealphase eine Verminderung der HF gegenüber der Follikelphase ab, meist begleitet von einer erhöhten LF und einem erhöhten LF/HF-Verhältnis. Zudem war im Orthostasetest das LF/ HF-Verhältnis in der Menstrual-, Lutealund Prämenstrualphase deutlich gesteigert, nicht aber in der Follikel- oder Ovulationsphase [23], was für eine stärkere adrenerge Aktivität in der Menstrual-, Luteal- und Prämenstrualphase spricht. Die verminderte HF als Ausdruck einer verminderten parasympathischen Aktivität spricht ebenfalls für eine sympathische Prädominanz in der Lutealphase. Demnach nimmt mit zunehmendem Östrogenspiegel in der Follikelphase die vagale Aktivität zum Herzen hin zu. Eine positive Korrelation zwischen der HF und Östrogenen zeigte sich auch in Bezug auf den Östrogenpeak im Serum bei Frauen nach Ovulationsinduktion [30].

\section{》) Postmenopausal verschiebt sich das sympathovagale Gleichgewicht zugunsten des Sympathikus}

Die Aktivität des ANS ändert sich mit der Menopause. Dies zeigen verschiedene Studien, welche die HRV vor und nach der Menopause verglichen [3, 13, 17, 20]. Bei postmenopausalen Frauen fand eine Verschiebung des sympathovagalen Gleichgewichts zugunsten des Sympathikus statt. Nebst einem erhöhten LF/HF-
Verhältnis äußerte sich dies in reduzierten TP- und HF-Werten [3, 13, 17, 20]. Dass hauptsächlich ovarielle Hormone an dieser Dysbalance beteiligt sind, zeigte eine weitere Studie, welche die HRV bei Frauen vor und nach einer bilateralen Ovarektomie verglich [16]. Die chirurgische Menopause bewirkte eine verminderte vagale Modulation der autonomen kardialen Kontrolle und folglich eine sympathische Hyperaktivität [16]. Mit dem Wegfallen der ovariellen Hormone sank die HF und das LF/HF-Verhältnis stieg an. Dies spricht für eine vermehrte sympathische Aktivität [16]. Die Messung wurde nach einer 3-monatigen transdermalen Östrogentherapie wiederholt. Unter der Behandlung zeigte sich eine Rückkehr der HRV auf präoperative Werte [16].

\section{Herzfrequenzvariabilität und menopausale Beschwerden}

Hitzewallungen zählen zu den häufigsten menopausalen Beschwerden. Aktuelle Studien fanden Unterschiede in der HRV zwischen symptomatischen und asymptomatischen postmenopausalen Frauen. Diese Beobachtung ist insofern von Interesse, als symptomatische gegenüber asymptomatischen Frauen ein höheres kardiovaskuläres Risiko aufweisen [6]. Kurzzeit-HRV-Messungen bei symptomatischen postmenopausalen Frauen zeigten bei einem erhöhten LF/HF-Verhältnis eine verringerte parasympathische Aktivität. Ferner nahm das LF/HF-Verhältnis mit zunehmendem Schweregrad der Symptome zu [11]. Beim gleichzeitigen Monitoring von Hitzewallungen und HRV kam es etwa 1 min vor Beginn der Hitzewallung zu einem abrupten HF-Abfall, der dann für einige Minuten auf erniedrigtem Niveau persistierte [28]. In der Folge war das LF/HF-Verhältnis erhöht [27]. Gemäß einer aktuellen Hypothese könnte eine sympathische Aktivierung als Auslöser von Hitzewallungen wirken [5].

\section{Einfluss einer Hormon- ersatztherapie auf die Herzfrequenzvariabilität}

Der Einfluss einer HRT auf die HRV hängt stark vom HRT-Typ ab. So vermochte eine 3-monatige transdermale HRT mit $50 \mu \mathrm{g}$
Östradiol $\left(\mathrm{E}_{2}\right)$ pro Tag bei ovarektomierten Frauen eine Anhebung der HRV auf präoperative Werte zu bewirken [16]. Die tägliche orale Einnahme von 0,625 mg konjugierten Östrogenen (CEE) hatte bei hysterektomierten Frauen ähnlich vorteilhafte Effekte auf die HRV, gekennzeichnet durch eine höhere HFnu, tiefere LFnu und ein tieferes LF/HF-Verhältnis im Vergleich zu Frauen ohne HRT [18].

Wird eine Östrogentherapie jedoch mit einem Gestagen kombiniert, ist die Datenlage widersprüchlich. Bei der Anwendung einer oralen kontinuierlichkombinierten HRT [CEE 0,625 mg/Tag und Medroxyprogesteronacetat (MPA) 2,5 mg/Tag] zeigte sich bis auf eine erhöhte RMSSD keine signifikante Veränderung der HRV-Parameter im Vergleich zum Zustand vor der Behandlung [7]. Im Head-to-head-Vergleich einer 3-monatigen oralen kontinuierlich-kombinierten HRT ( $E_{2} 2 \mathrm{mg} / \mathrm{Tag}$ und MPA 2,5 mg/ Tag) mit einer Östrogenmonotherapie ( $\mathrm{E}_{2} 2 \mathrm{mg} / \mathrm{Tag}$ ) war die HF bei Frauen mit einer Kombinationstherapie erhöht [4]. Allerdings fanden Lantto et al. [10] unter einer 6-monatigen Kombinationstherapie vermehrt supraventrikuläre Extrasystolen bei symptomatischen Frauen. Eine 6-monatige orale kontinuierlich-kombinierte HRT mit einem Nortestosteronderivat $\left(\mathrm{E}_{2} 2 \mathrm{mg} /\right.$ Tag und Norethisteron $1 \mathrm{mg} /$ Tag) bewirkte zwar einen Abfall der HF, das LF/HF-Verhältnis zeigte jedoch keine Veränderung. Daraus schlossen die Autoren, dass eine HRT mit einem Nortestosteronderivat die HRV nicht wesentlich beeinflusst [19].

\section{Fazit für die Praxis}

- Die ovariellen Steroidhormone Östrogen und Progesteron scheinen das autonome Gleichgewicht am Herzen zu beeinflussen.

- Während des Menstruationszyklus zeigt die parasympathische Aktivität eine positive Korrelation mit dem Östrogenserumspiegel.

- Nach der Menopause ist ein Abfall von parasympathischen Parametern zu beobachten. Diese Veränderungen können teilweise durch eine HRT kompensiert werden, wobei die 
Datenlage zu verschiedenen HRT-Typen kontrovers ist.

- Die Kombination mit einem Gestagen kann die vorteilhafte Wirkung der Östrogene aufheben oder sogar den Sympathikotonus erhöhen. Es besteht demnach ein Zusammenhang zwischen der HRV und der endo- und exogenen Östrogenexposition.

- Ein verminderter parasympathischer "Drive", der mit einem postmenopausalen Östrogenmangel einhergeht, trägt auch zu einem erhöhten KHK-Risiko bei postmenopausalen Frauen bei. Insbesondere Frauen mit menopausalen Beschwerden scheinen davon betroffen zu sein.

\section{Korrespondenzadresse}

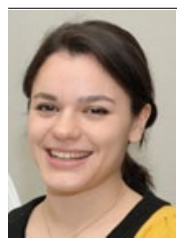

\section{G. Capaldo}

Abteilung für Gynäkologische

Endokrinologie und Reproduktionsmedizin, Frauenklinik, Inselspital Bern Effingerstr. 102, 3010 Bern Schweiz giuliana.capaldo@ students.unibe.ch

Interessenkonflikt. Die korrespondierende Autorin gibt für sich und ihre Koautoren an, dass kein Interessenkonflikt besteht.

\section{Literatur}

1. Bai X, Li J, Zhou L et al (2009) Influence of the menstrual cycle on nonlinear properties of heart rate variability in young women. Am J Physiol Heart Circ Physiol 297:H765-H774

2. Berntson GG, Bigger JT Jr, Eckberg DL et al (1997) Heart rate variability: origins, methods, and interpretive caveats. Psychophysiology 34:623-648

3. Davy KP, Desouza CA, Jones PP et al (1998) Elevated heart rate variability in physically active young and older adult women. Clin Sci 94:579-584

4. Farag NH, Nelesen RA, Parry BL et al (2002) Autonomic and cardiovascular function in postmenopausal women: the effects of estrogen versus combination therapy. Am J Obstet Gynecol 186:954961

5. Freedman RR, Kruger ML, Wasson SL (2011) Heart rate variability in menopausal hot flashes during sleep. Menopause 18:897-900

6. Gambacciani M, Pepe A (2009) Vasomotor symptoms and cardiovascular risk. Climacteric 12(Suppl 1):32-35

7. Gokce M, Karahan B, Yilmaz R et al (2005) Long term effects of hormone replacement therapy on heart rate variability, QT interval, QT dispersion and frequencies of arrhythmia. Int J Cardiol 99:373-379

Gynäkologische Endokrinologie 2013 • 11:140-143 DOI 10.1007/s10304-013-0553-7

(c) Springer-Verlag Berlin Heidelberg 2013

\section{G. Capaldo $\cdot$ M. Wilhelm · P. Stute}

\section{Herzfrequenzvariabilität in der Postmenopause}

\section{Zusammenfassung}

Autonomes Nervensystem und Herzfrequenzvariabilität. Sowohl eine Dysregulation des autonomen Nervensystems (ANS) als auch die Menopause sind mit einem erhöhten kardiovaskulären Risiko verbunden. Das kardiale ANS kann anhand der Herzfrequenzvariabilität (HRV) beurteilt und in Frequenzspektren aufgespalten werden, die sich dem Parasympathikus- und/oder Sympathikuseinfluss zuordnen lassen.

Einfluss der endokrinen Lebensphasen. Bereits während des Menstruationszyklus zeigt sich eine zyklusabhängige Fluktuation der HRV. Mit dem menopausalen Östrogenabfall ist ein Anstieg des Sympathikoto- nus mit HRV-Reduktion verbunden, was mit einem erhöhten kardiovaskulären Risiko einhergeht. Das mit menopausalen Hitzewallungen verbundene erhöhte kardiovaskuläre Erkrankungsrisiko ist möglicherweise ebenso auf eine Sympathikusaktivierung zurückzuführen.

Therapie. Eine Hormonersatztherapie vermag eventuell eine Wiederherstellung der autonomen Balance herbeizuführen.

Schlüsselwörter

Autonomes Nervensystem - Menopause . Hitzewallungen $\cdot$ Hormonersatztherapie Östrogene

\section{Heart rate variability in postmenopause}

\section{Abstract}

Autonomic nervous system and heart rate variability. Both dysregulation of the autonomic nervous system (ANS) and menopause are associated with a higher cardiovascular risk. The cardiac ANS can be assessed by means of heart rate variability (HRV), as the variation between consecutive RR intervals is influenced by parasympathetic and sympathetic mediators.

Effects of menstrual cycle phases. During the menstrual cycle, a cycle-dependent HRV fluctuation has been found, and a HRV reduction occurs upon estrogen withdrawal after menopause. Postmenopausal HRV reduction is associated with a higher sympathetic tone and is accompanied by a higher cardiovascu- lar risk. Recent findings suggest a role of the sympathetic drive in the genesis of menopausal hot flashes, as an abrupt reduction of the parasympathetic tone with a predominance of the sympathetic tone occurs during a hot flash.

Therapy. Hormone replacement therapy may restore the autonomic balance. However, type and combination of hormones may be crucial.

\section{Keywords}

Autonomic nervous system - Menopause . Hot flashes · Hormone replacement therapy . Estrogens
8. Hulley S, Grady D, Bush T et al (1998) Randomized trial of estrogen plus progestin for secondary prevention of coronary heart disease in postmenopausal women. Heart and Estrogen/progestin Replacement Study (HERS) research group. JAMA 280:605-613

9. Kannel WB, Hjortland MC, McNamara PM et al (1976) Menopause and risk of cardiovascular disease: the Framingham study. Ann Intern Med 85:447-452

10. Lantto H, Haapalahti P, Tuomikoski P et al (2012) Vasomotor hot flashes and heart rate variability: a placebo-controlled trial of postmenopausal hormone therapy. Menopause 19:82-88

11. Lee JO, Kang SG, Kim SH et al (2011) The relationship between menopausal symptoms and heart rate variability in middle aged women. Korean J Fam Med 32:299-305

12. Liao D, Cai J, Rosamond WD et al (1997) Cardiac autonomic function and incident coronary heart disease: a population-based case-cohort study. The ARIC study. Atherosclerosis risk in communities study. Am J Epidemiol 145:696-706
13. Liu CC, Kuo TB, Yang CC (2003) Effects of estrogen on gender-related autonomic differences in humans. Am J Physiol Heart Circ Physiol 285:H2188H2193

14. Mendelsohn ME, Karas RH (2005) Molecular and cellular basis of cardiovascular gender differences. Science 308:1583-1587

15. Mendelsohn ME, Karas RH (1999) The protective effects of estrogen on the cardiovascular system. N Engl J Med 340:1801-1811

16. Mercuro G, Podda A, Pitzalis L et al (2000) Evidence of a role of endogenous estrogen in the modulation of autonomic nervous system. Am J Cardiol 85:787-789, A789

17. Moodithaya SS, Avadhany ST (2009) Comparison of cardiac autonomic activity between pre and post menopausal women using heart rate variability. Indian J Physiol Pharmacol 53:227-234

18. Neves VF, Silva de Sá MF, Gallo L Jr et al (2007) Autonomic modulation of heart rate of young and postmenopausal women undergoing estrogen therapy. Braz J Med Biol Res 40:491-499 
19. Niskanen L, Laitinen T, Tuppurainen M et al (2002) Does postmenopausal hormone replacement therapy affect cardiac autonomic regulation in osteoporotic women? Menopause 9:52-57

20. Ribeiro TF, Azevedo GD, Crescencio JC et al (2001) Heart rate variability under resting conditions in postmenopausal and young women. Braz J Med Biol Res 34:871-877

21. Rosano GM, Chierchia SL, Leonardo F et al (1996) Cardioprotective effects of ovarian hormones. Eur Heart J 17(Suppl D):15-19

22. Rosano GM, Leonardo F, Sarrel PM et al (1996) Cyclical variation in paroxysmal supraventricular tachycardia in women. Lancet 347:786-788

23. Saeki Y, Atogami F, Takahashi K et al (1997) Reflex control of autonomic function induced by posture change during the menstrual cycle. J Auton Nerv Syst 66:69-74

24. Sato N, Miyake S, Akatsu J et al (1995) Power spectral analysis of heart rate variability in healthy young women during the normal menstrual cycle. Psychosom Med 57:331-335

25. Stauss HM (2003) Heart rate variability. Am J Physiol Regul Integr Comp Physiol 285:R927-R931

26. Task Force of the European Society of Cardiology and the North American Society of Pacing and Electrophysiology (1996) Heart rate variability. Standards of measurement, physiological interpretation, and clinical use. Eur Heart J 17:354-381

27. Thurston RC, Christie IC, Matthews KA (2012) Hot flashes and cardiac vagal control during women's daily lives. Menopause 19:406-412

28. Thurston RC, Christie IC, Matthews KA (2010) Hot flashes and cardiac vagal control: a link to cardiovascular risk? Menopause 17:456-461

29. Tsuji H, Larson MG, Venditti FJ Jr et al (1996) Impact of reduced heart rate variability on risk for cardiac events. The Framingham heart study. Circulation 94:2850-2855

30. Weissman A, Lowenstein L, Tal J et al (2009) Modulation of heart rate variability by estrogen in young women undergoing induction of ovulation. Eur J Appl Physiol 105:381-386

31. Yildirir A, Kabakci G, Akgul E et al (2002) Effects of menstrual cycle on cardiac autonomic innervation as assessed by heart rate variability. Ann Noninvasive Electrocardiol 7:60-63

\section{Gestationsdiabetes}

Ab dem 03.03.2012 hat der Gesetzgeber im Rahmen der Mutterschaftsrichtlinie ein verbindliches Vorgehen für Screening und Diagnostik auf Gestationsdiabetes für alle Schwangeren eingeführt. Ziel des ärtzlichen Vorgehens nach der Diagnose ist eine individuelle, risikoangepasste Intensität von Überwachung und Therapie.

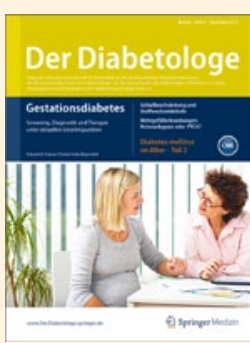

Das Schwerpunktheft ,Gestationsdiabetes" von Der Diabetologe (Ausgabe 8/2012) beleuchtet in folgenden Beiträgen Gegenwart und Zukunft der Erkrankung:

- Screening und Diagnostik des Gestationsdiabetes

- Diabetologisches und geburtshilfliches Management des Gestationsdiabetes

- "Vitamin D and Lifestyle Intervention for Gestational Diabetes Mellitus Prevention" (DALI)

- Hyperglycemia and Adverse Pregnancy Outcome Study

Bestellen Sie diese Ausgabe zum Preis von 35,- EUR zzgl. Versandkosten bei Springer Customer Service Center Kundenservice Zeitschriften

Haberstr. 7

69126 Heidelberg

Tel.: +49 6221-345-4303

Fax: +49 6221-345-4229

E-Mail: leserservice@springer.com

Suchen Sie noch mehr zum Thema? Mit e.Med, dem Online-Paket von Springer Medizin, können Sie schnell und komfortabel in über 500 medizinischen Fachzeitschriften recherchieren.

Weitere Infos unter springermedizin.de/eMed.

\section{Arzt-Patienten-Verhältnis}

Reflexionen über das Arzt-Patienten-Verhältnis sind vermutlich ebenso alt wie die Medizin selbst. Mit den Veränderungen der Gesundheitsversorgung definiert sich naturgemäß auch die Rolle von Arzt und Patient immer

wieder neu. Die

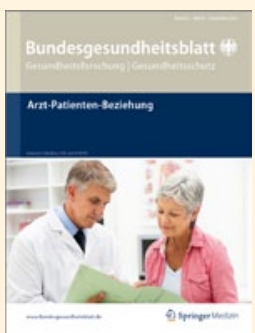

Ausgabe 9/2012 der Zeitschrift Bundesgesundheitsblatt befasst sich ausführlich mit den aktuellen Entwicklungen. Das Heft greift die Aufgabe auf, dass das Verhältnis zwischen Arzt und Patient in der Gegenwart multidisziplinär - medizinisch, kulturwissenschaftlich, ethisch, rechtswissenschaftlich - reflektiert werden sollte.

Das Schwerpunktheft „Arzt-Patienten-Verhältnis" beinhaltet u.a. folgende Beiträge:

- Patientenautonomie und Patientenrechte

- Die ärztliche Rolle im Wandel

- Placeboaspekte der Arzt-Patienten-Beziehung

- Geschlechter(rollen)-aspekte in der Arzt-Patient-Interaktion

- Der Arzt als Begleiter in der Palliativmedizin

- Die Rolle der Pflege im Gesundheitswesen

- Interkulturelle Aspekte der Interaktion und Kommunikation im psychiatrisch/psychotherapeutischen Behandlungssetting

Bestellen Sie diese Ausgabe zum Preis von EUR 14,00 zzgl. Versandkosten bei:

Springer Customer Service Center GmbH Kundenservice Zeitschriften

Haberstr. 7

69126 Heidelberg

Tel.: +49 6221-345-4303

Fax: +49 6221-345-4229

leserservice@springer.com

Suchen Sie noch mehr zum Thema? Mit e.Med, dem Online-Paket von Springer Medizin, können Sie schnell und komfortabel in über 500 medizinischen Fachzeitschriften recherchieren.

Weitere Infos unter springermedizin.de/e.Med 